\title{
ERRATUM
}

\section{Electronics based on two-dimensional materials}

Gianluca Fiori, Francesco Bonaccorso, Giuseppe lannaccone, Tomás Palacios, Daniel Neumaier, Alan Seabaugh, Sanjay K. Banerjee and Luigi Colombo

Nature Nanotechnology 9, 768-779 (2014); published online 6 October 2014; corrected after print 5 November 2014.

In the version of this Review Article originally published, in Fig. 4c, the $y$-axis values were incorrect; they should have been ' $10^{\circ}, 10^{2}, 10^{4}$ and $10^{6}$ (from bottom to top). This error has now been corrected in the online versions of the Review Article. 